\title{
KASUISTIKEN
}

Klin. Pädiat. 196 (1984) 44-46

(C) F. Enke Verlag Stuttgart

\section{Fibrinpleurodese eines beidseitigen Spannungspneumothorax bei Mukoviszidose}

\author{
H. Segerer*, K. Richter*, J. Scheele** \\ - Kinderklinik mit Poliklinik der Universität Erlangen-Nürnberg (Direktor: Prof. Dr. K. Stehr) \\ * Chirurgische Klinik mit Poliklinik der Universität Erlangen-Nürnberg (Direktor: Prof. Dr. F.P. Gall)
}

\section{Zusammenfassung}

Ein beidseitiger rezidivierter Spannungspenumothorax bei einer 18jährigen Patientin mit Mukoviszidose wurde durch Fibrin-Pleurodese behandelt. Innerhalb des 20 monatigen Beobachtungszeitraumes ist kein Rezidiv aufgetreten.

Fibrin Glue Pleurodesis of a Bilateral Tension Pneumothorax in Cystic Fibrosis

A bilateral recurrent tension pneumothorax in an 18-yearold patient with cystic fibrosis was treated by fibrin glue pleurodesis. Within the observation period of 20 months no relapse has occurred.

\section{Einführung}

Mit zunehmender Lebenserwartung der Patienten mit Mukoviszidose in den letzten Jahren nimmt die Komplikation eines lebensbedrohenden Spannungspneumothorax an Häufigkeit zu $(1,5,8,14)$.

Wir konnten eine Patientin mit zystischer Fibrose und rezidivierendem Spannungspneumothorax durch Fibrinpleurodese eines beidseitigen Spannungspneumothorax erfolgreich behandeln.
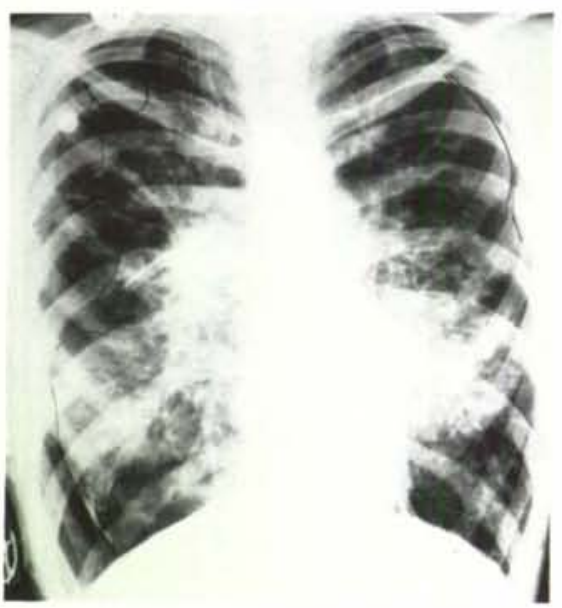

Abb. 1 Beidseitiger Spannungspneumothorax bei Mukoviszidose. Röntgenaufnahme bei Aufnahme ( $\longrightarrow$ Lungengrenzen).

\section{Fallbericht}

Bei Angelika H. (KB.-Nr. 318 204) wurde im Alter von 10 Jahren eine Mukoviszidose festgestellt. Mit 16 Jahren bestanden ausgedehnte Bronchiektasen. Im Verlauf der nächsten 2 Jahre traten wenigstens drei Episoden einseitiger Pneumothoraces auf, die konservativ behandelt werden konnten.

Eine vierte Episode kurz nach ihrem 18. Geburtstag begann mit heftiger Atemnot und stechenden beidseitigen Thoraxschmerzen.

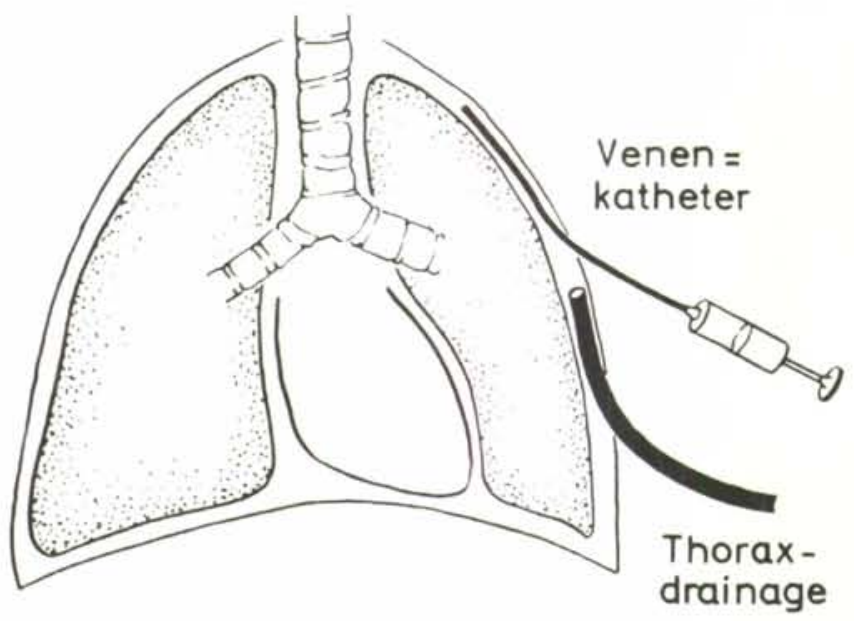

Abb. 2a Schemadarstellung: Bei liegender Thoraxsaugung werden Cava-Katheter in den Pleuraspalt eingelegt.

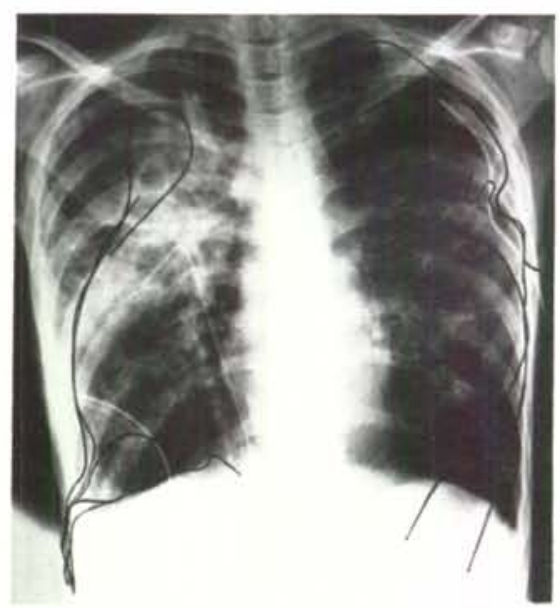

Abb. $2 \mathrm{~b}$ Thoraxsaugungen und $(\longrightarrow$ Cava-Katheter in situ. 


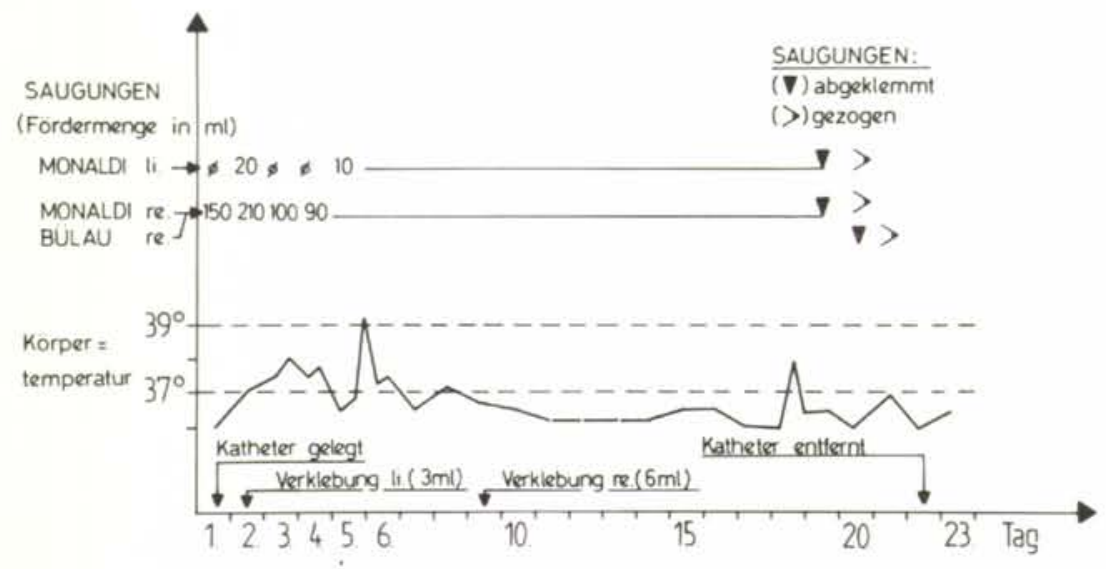

Abb. 3 Verlaufskurve der Pat. A.H.: Kein Pleurareizerguß, keine Temperaturerhöhung im Zusammenhang mit den Fibrinklebungen am 2. und 9. Behandlungstag.
Nach der Diagnose eines rechtsseitigen Pneumothorax in einem auswärtigen Krankenhaus wurden zwei Drainagen rechts angelegt. Dennoch persistierte die Atemnot; das Mädchen wurde zu uns verlegt. Hier stellte sich ein beidseitiger Spannungspneumothorax heraus; die Atemnot konnte erst nach Lagekorrektur der beiden Saugungen und Anlegen einer dritten gebessert werden (Abb. 1).

Bei noch unvollständig expandierten Lungen wurden auf beiden Seiten unter Durchleuchtungskontrolle mehrere Cava-Katheter in den Pleuraspalt eingelegt (Abb, 2).

Am 2. Behandlungstag wurde über diese Katheter $3 \mathrm{ml}$ Fibrinkleber* in den Pleuraspalt der linken Thoraxseite instilliert.

Wegen anfangs außerordentlich großer Lecks der rechten Lunge konnte die Fibrinpleurodese auf dieser Seite erst am 9. Behandlungstag durch Einspritzen von $6 \mathrm{ml}$ Fibrinkleber-Gemisch erfolgen (Abb. 3).

Am 10. Tag nach der zweiten Verklebung wurden beide MonaldiSaugungen abgeklemmt und 24 Stunden später gezogen. Einen Tag später konnte auch die Bülau-Drainage entfernt werden. Die Reexpansion beider Lungen blieb erhalten; seit nunmehr 20 Monaten ist das Mädchen rezidivfrei ( $\mathrm{Abb}$. 4). Es ist arbeitsfähig und mäßjger sportlicher Belastung (Skilauf) gewachsen.

* Fibrinkleber Human Immuno, Immuno AG, Wien/Österreich, vermischt mit Thrombin, Faktor XIII und $\mathrm{Ca}^{2+}$-Ionen (vgl. 11).

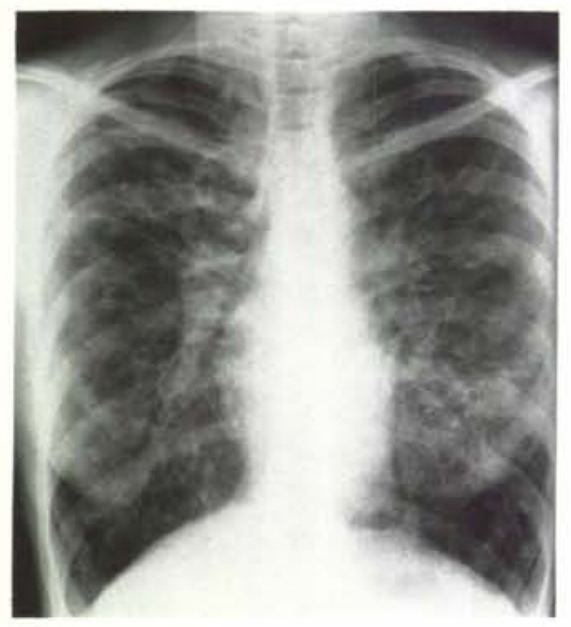

Abb. 4 Thorax-Kontrollaufnahme 20 Monate nach beidseitiger Fibrinpleurodese.
Schmerzen wurden beim Einspritzen des Fibrinklebers nicht angegebẻn; Entzündungszeichen traten nicht auf. Auch Pleurareizergüsse oder eine Temperaturerhöhung wurden nicht beobachtet.

\section{Diskussion}

Die Pleurodese durch Fibrinkleber wurde erstmals von Spängler (9) an der Pleura von Hunden erprobt. Scheele verwendete dieses Verfahren erfolgreich bei sieben Erwachsenen mit rezidivierendem oder persistierendem Pneumothorax (11).

Beide Autoren betonten die gute Verträglichkeit dieser Art der Pleurodese. Unsere Erfahrungen bestätigen diese $\mathrm{Be}$ richte, so daß uns diese Behandlungsmethode des rezidivierenden Spontanpneumothorax insbesondere bei der $\mathrm{Mu}$ koviszidose gegenüber Klebstoffen anderer Art oder Pleurairritantien überlegen erscheint.

Im Vergleich zu Silbernitrat (3), Talkpuder (2, 15), Quinacrin $(6,7,17)$, Tetrazyklinen $(10,13,16)$ oder Cyanoacrylat-Kleber (12) muß die Verzichtbarkeit einer MorphinAnalgese und das langanhaltend gute Ergebnis betont werden.

Die meist als definitive Therapie auch bei der Mukoviszidose empfohlene Thorakotomie mit partieller Pleurektomie bei rezidivierendem Auftreten eines Pneumothorax (4, 8, 14, 17) wäre wegen des schlechten Allgemeinzustandes unserer Patientin nicht möglich gewesen.

Das bislang sehr zufriedenstellende Ergebnis sollte anhand weiterer Beobachtungen überprüft werden.

\section{Literatur}

1 Paxton D.G., E.P. Scott: Pneumothorax: An Unusual Complication in Fibrocystic Disease. Am. J. Dis. Child. 111 (1966) $311-312$

2 Adler, R.H.: A Talc Powder Aerosol Method for the Prevention of Recurrent Spontaneous Pneumothorax. Ann. Thorac. Surg. 5 (1968) 474-477

3 Anderson, J., H. Nissen: Results of Silver Nitrate Pleurodesis in Spontaneous Pneumothorax. Dis. Chest. 54 (1968) 230-233

4 Lifschitz, M.I., F.O. Bowman jr., C.R. Denning, R.H. Wylie: Pneumothorax as a Complication of Cystic Fibrosis. Am. J. Dis. Child 116 (1968) 633-640 
5 Boat, T.F., P.A. di Sant'Agnese, W.J. Warren, S.A. Handwerger: Pneumothorax in Cystic Fibrosis. J.A.M.A. 209 (1969) 14981504

6 Cattaneo, S. M., H.D. Sirak, K.P. Klassen: Recurrent spontaneous pneumothorax in the high-risk patient. Management with intrapleural quinacrine. J. Thorax Cardiovasc. Surg. 66 (1973) 467-471

7 Kattwinkel, J., L.M. Taussig, C.L. McIntosh, P.A. di Sant'Agnese, T.F. Boat, R.E. Wood: Intrapleural instillation of quinacrine for recurrent pneumothorax. J.A.M.A. 226 (1973) 557

8 Stowe, S.M., T.F. Boat, H. Mendelsohn, R.C. Stern, A.S. Tucker, C.F. Doershuk, L.W. Matthews: Open Thoracotomy for Pneumothorax in Cystic Fibrosis. American Rev. Resp. Dis. 111 (1975) 611-617

9 Spängler, H.P.: Gewebeklebung und lokale Blutstillung mit Fibrinogen, Thormbin und Blutgerinnungsfaktor XIIl. Wiener klin. Wochenschr. 88, Suppl. 49 (1976) 3-18

10 Luck, S.R., J.G. Raffensperger, H.J. Sullivan, L.E. Gibson: Management of pneumothorax in children with chronic pulmonary disease. J. Thorac. Cardiovasc. Surg. 74 (1977) $834-$ 839
11 Scheele, J., E. Mühe, F. Wopfner: Fibrinklebung bei Spontanpneumothorax. Chirurg 49 (1978) 236-243

12 Takeno, $Y$.: New treatment of spontaneous pneumothorax by liquid glue nebulization. Bronchopneumologie 28 (1978) 1928

13 Goldszer, R.C., J. Bennet, J. VanCampen, J. Rudnitzky: Intrapleural Tetracycline for Spontaneous Pneumothorax. J.A.M.A. 241 (1979) 724-725

14 Knight, R.K., J.C. Batten: Pneumothorax in Cystic Fibrosis. In: Perspectives in Cystic Fibrosis. Proceedings of the 8th international cystic fibrosis congress held in Toronto, Canada, May 26-30, 1980. Edited by Jennifer M. Sturgers. Published by the Canadian Cystic Fibrosis Foundation

15 Nandi, P.: Recurrent Spontaneous Pneumothorax: An Effective Method of Talc Poudrage. Chest 77 (1980) 493-495

16 Cannon, W.B., J.B.D. Mark, R.W. Jamplis: Pneumothorax: A Therapeutic Update. Am. J. Surg. 142 (1981) 26-29

17 McLaughlin, F.J., W.J. Matthews jr., D.J. Strieder, K.T. Khaw, S. Schuster, H. Shwachman: Pneumothorax in cystic fibrosis: Management and outcome. J. Pediatrics 100 (1982) 863-869

Dr. H. Segerer, Univ.-Kinderklinik, Loschgestraße 15, D-8520 Erlangen 